\title{
De las Representaciones Discursivas AL ORDEN DEL DisCURSO: Una Perspectiva Discursiva de La INTERVENCIÓN DEL TRABajo SOCIAL ${ }^{*}$
}

Por: Leonardo Oneto Piazze

Artículo Recibido: 25 de enero de 2007

Artículo Aceptado: 15 de marzo de 2007

\section{RESUMEN}

Esta reflexión sobre los fundamentos de la acción profesional representa un intento de describir una forma de entender la intervención del Trabajo Social, definiéndola desde una aproximación de construcción social. Esta perspectiva se auxilia de una comprensión dialéctica para describir la relación entre los procesos materiales y simbólicos, como también remite a la aproximación arqueológica de Foucault ${ }^{1}$ y la pragmática discursiva contemporánea en su versión crítica. ${ }^{2}$

Palabras Claves: Discurso, intervención social, representaciones, dimensión ético/política, interpretación.

\section{ABSTRACT}

This reflection about the basis of the professional action represents an attempt to describe a way of understanding the Social Work intervention, defining it from a social construction approximation. This perspective is supported on a dialectical comprehension to describe the relationship between the symbolic and material processes, as well as it refers to the Foucault's $s^{1}$ archeological approximation and to the contemporary discursive pragmatics in its critical version. ${ }^{2}$

Key Words: Discursive representation, discourse, intervention, Social Work, symbolic world, dialectics, political/ethical dimension.

Palobrano. 8 Agosto/2007 


\section{Introducción}

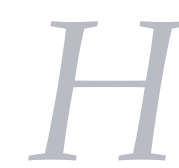

istóricamente, los fundamentos de la acción transformadora en Trabajo Social han estado sustentados en las teorías y valores dominantes en cada momento, y en menor medida en la posible evolución de los "objetos" específicos de esta acción, sea cual sea la forma de organizar o definir la situación o fenómeno problemático por parte de la estructura institucional. En otras palabras, las formas de organizar y definir el fenómeno tributan centralmente a las ópticas desde las cuales se ha aprendido en cada época a leer esa "realidad", lo que no significa que la sociedad no se haya complejizado y especializado crecientemente, sino, que esas mismas transformaciones dependen de la posibilidad misma de ser nombradas, y sólo desde sí mismas.

Por tanto, lo que ha sido "razonable" como fundamento de la acción en cada período histórico y hoy mismo, refiere a los diferentes modos de entender las

3 Molitor, Michel. Le hermenéutique collective, en "Méthodes d'analyse de contenu et sociologie". Facultés Universitaires SaintLouis. Bruxelles, 1990. P. $23-31$.-

4 Hablaremos de lo "problemático social" como una manera de reconocer el carácter histórico y social de su construcción. Se pasa del fenómeno a lo problemático a partir de la progresiva "fusión de horizontes"* de múltiples actores en un orden discursivo en que el poder está distribuido normalmente de manera desigual. $\left(^{*}\right)$ "Fusión de horizontes" en el sentido que le da Hans Georg Gadamer en Verdad y Método I y II, 1960 como "entenderse con otro sobre algo".

5 Ver, Touraine, Alain: 1993. Crítica a la modernidad. FCE. México. "prácticas transformadoras de lo social" dado un modelo cultural particular colonizado por las explicaciones "naturalizadas" en cada momento.

Por lo señalado, esta propuesta va a ubicar la intervención profesional al interior de lo que Michel Foucault denominó orden discursivo, asumiendo lo heterogéneo y complejo de las relaciones y condiciones de como los discursos se desarrollan y sustentan socio - políticamente.

Al interior de la presencia de un orden discursivo se discutirá acerca de la relación dialéctica entre los procesos materiales /estructurales y los simbólicos. Con este fin se transitará a través de los conceptos de representaciones sociales, distinguiendo modelos, estructuras y esquemas de representación y sus condicionantes estructurales como los reconoce la versión de M. Molitor de "Hermenéutica Colectiva" ${ }^{3}$. A partir de estos se analizará los procesos de intervención en la dialéctica de conformación de discursos sobre lo problemático social ${ }^{4}$, considerando el aporte del Análisis Crítico del Discurso, para proceder al análisis de las condiciones de posibilidad de su transformación.

Finalmente el texto propone la consideración de un eje ético/político como articulador de la intervención en una progresión que involucra el orden del discurso, las representaciones y la conformación de actoría social. ${ }^{5}$ 


\section{La Intervención como Acción sobre el Mundo Simbólico: La Construcción de Representaciones}

En una segunda perspectiva, esta vez ya no desde el orden discursivo mismo, sino desde los sujetos, ${ }^{6}$ podemos apreciar que cualquiera que sea el nivel de intervención, los sujetos están más o menos conscientes de su posición en la estructura social y de sus relaciones sociales. La referencia a sí mismo, al otro y al nosotros como perspectiva relacional marca un vector de construcción de realidad en que se definen las identidades y las vinculaciones singulares con el entorno social.

Una aproximación a la compresión de los sujetos implica, la dilucidación de la apropiación de lo "real" desde sus propias biografías, así como, la apropiación social, en tanto, cuanto la biografía individual esta marcada por su ser social. Esto significa que la asimilación particular de "lo real" esta referida, a su vez al universo social del que se forma parte, como a la historia personal.

Así, a modo de ilustración, si damos una mirada a la figura del "robo". Las personas van a leer distinto este fenómeno dadas las mediaciones de ubicación en las relaciones sociales. Un niño nacido en una cultura criminógena entenderá que esto es natural, un adulto en esta cultura dirá que, si no hay otra forma de subsistir es lícito robar a quien tiene mucho. Un empresario dirá que es lícito no pagar los impuestos dado que estos son una injusticia, una expropiación estatal de lo ganado con esfuerzo, más aún, cuando estos son usados por gobiernos que no administran bien los recursos. Este podrá incluso pensar que es bueno para el desarrollo del país no pagarlos en su totalidad. Por su parte, un obrero podría pensar que es lícito sacar algún material de la fábrica porque no se siente adecuadamente retribuido y no se identifica con la empresa y sus resultados.

Pero, si el padre de nuestro sujeto ha sido un hombre que aprendió que lo ajeno no se toca, y transmitió estos valores de respeto a la propiedad ajena, sea cual fuere su posición en la estructura social, patrón u obrero tenderá a mantener esa actitud salvo que hayan mediado otras influencias en su socialización. Lo que se señala es que se es socializado en y desde una posición social, en y desde una biografía personal y familiar, de este modo, en el último caso tendería a primar la biografía por sobre la posición. Estas consideraciones muestran lo eventual que pueden ser los procesos de construcción de estructuras y esquemas de interpretación de lo real.

Estos quiebres respecto al fenómeno, dicen relación a la reinterpretación que hace

6 Puede aparecer contradictorio la apelación al sujeto en relación al orden discursivo si consideramos que Foucault proclama la muerte del sujeto en el discurso, dado que lo dicho esta ya dicho socialmente. En este sentido asumimos la categoría de orden discursivo de manera dialéctica y en tensión, asumiendo quiebres entre los discursos hegemónicos y los particulares situados, lo que hace a la posibilidad de su transformación. 
${ }^{7}$ Molitor, Michel.

8 En el sentido del Wittgenstein de los años 30 .

9 Oneto, Leonardo (2003). "La Representación de la Pobreza en los Textos de los Centros de Estudio del Chile de los Noventa". Tesis para optar al Grado de Doctor en Lingüística de la Facultad de Filosofía y Educación. Instituto de Literatura y Ciencias del Lenguaje de la Universidad Católica de Valparaíso. el sujeto desde lo biográfico respecto las relaciones sociales entendidas en un sentido más estructural. Aunque todos los sujetos dirán y aceptarán que es malo robar como modelo de interpretación social, ${ }^{7}$ cada uno lo lee desde su propia red de relaciones y desde su propia historia personal ${ }^{8}$ y social, así, un mismo referente cultural asume distintas connotaciones según la posición de los sujetos en la estructura, y a medida que nos aproximamos a grupos particulares los "quiebres" se multiplican llegando a negar ocasionalmente la determinación estructural.

Cada sujeto individualmente considerado, como constructor de significados a partir de redes de conversaciones constituirá "juegos de lenguaje"9 aceptados por determinadas comunidades de habla o circunstancias. En diversos círculos son válidas ciertas maneras de decir, ciertas formas de valorar. Cada uno se relaciona desde un libreto particular en que lo biográfico explica la forma como se asimilan los acontecimientos a partir de un mundo social compartido, desde una posición en la estructura. En todo caso, será la situación, la contingencia, el lugar desde el que se configura la significación, el factor determinante de su comprensión.

Hemos señalado que se construye "lo real social" en referencia a una posición en la estructuración de relaciones respecto a otros. La acción profesional tiene en este nudo de significaciones un enorme desafío, dado que normalmente la construcción de la intervención respecto a lo "social problematizado" surge de una negociación permanente de significaciones presentes en los múltiples actores individuales, institucionales y organizacionales implicados. Por ejemplo, el sentido que posee la participación de los apoderados (o representantes de los alumnos) en las escuelas públicas puede ser visto desde el Ministerio de Educación como la constitución de espacios de diálogo para la construcción del proyecto educativo. Para los Directores de Escuela será un problema de procedimientos, de cómo implementar para que los padres y apoderados compartan tareas tan complejas siendo que poseen poca información, disponen de poco tiempo y son muy críticos. Por su parte, los padres y apoderados, tienen una visión a partir de la experiencia particular con cada "Profesor Jefe", donde el tema es el juicio sobre el grado en que éste estaría respondiendo a las expectativas que los padres tienen; que se les enseñe efectivamente a sus hijos, en último término, que los cuadernos reflejen un grado de avance en los contenidos parecido al de los colegios particulares (privados), y que los traten de manera digna.

Podríamos repetir este ejercicio en el área de salud con los programas de prevención de consumo de droga, considerando si los conceptos usados para 
persuadir respecto al no consumo se relacionan con lo que piensan los grupos de riesgo, o respecto al tipo de vivienda y barrio esperado por la gente versus la oferta estatal, o cualquier otro tipo de acción de las políticas sociales y nos encontraríamos con tramas parecidas de figuras quebradas. Es a partir de éstas que los Trabajadores Sociales tienen que organizar sus intervenciones para lograr "anclar" con los distintos referentes actoriales $y$ movilizar sus posibilidades desde una acción comunicativa que no puede sino ser situada.

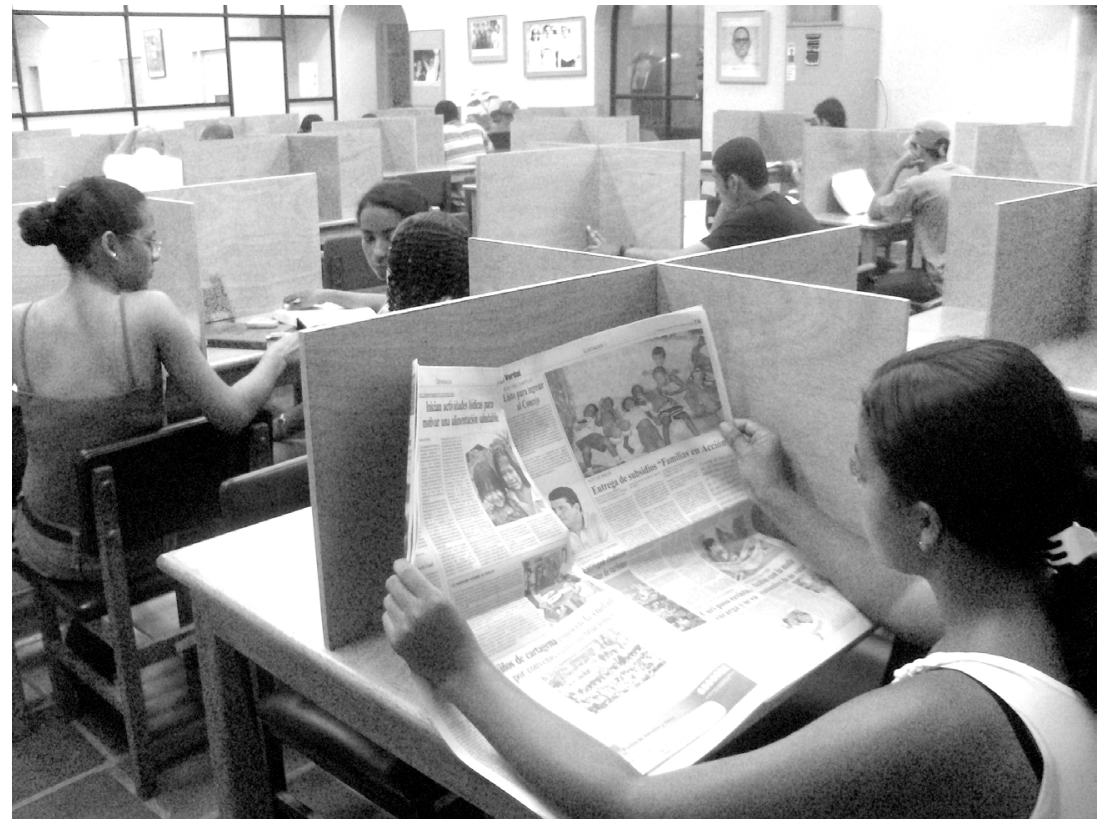

Foto: Nestor Castellar Acosta, Universidad de Cartagena

El supuesto de la acción discursiva es que las representaciones discursivas reflejan la organización de la interpretación de mundo que poseen los hablantes. Por ejemplo, comparada la representación discursiva de la pobreza a partir de las nominalizaciones realizada en un estudio comparativo en textos de derecha, izquierda y centro, ${ }^{10}$ se observa que el discurso de derecha usa más recurrentemente la palabra "pobres" para referir a los grupos en desventaja, mientras que el discurso de centro habla con más frecuencia de "grupos vulnerables"y los de izquierda de trabajadores o cesantes. Es evidente que estos discursos anclan con el universo ideológico desde el que provienen, la izquierda entiende la precariedad como bajos salarios o censantía puesto que su análisis de los grupos en desventaja es estructural. Cuando se revisa los verbos que refieren las acciones sobre la pobreza, se observa que el discurso de derecha no habla nunca de "superar la pobreza", mientras que el de izquierda habla de "superar" en un 36\% de las oportunidades. La acción verbal más frecuente en el discurso de derecha es ir a la "reducción" de la pobreza, mientras que en los de centro se usa más comúnmente ir a la "superación", acción verbal que mitiga la idea de que lo que hay que hacer es "superar" la pobreza y no tender a su "superación" como acción transitiva. De este modo, pensar en una estrategia discursiva para concertar las voluntades en torno a este tema implica encontrar una forma de nombrar y referir la acción que interprete un espectro diverso, sabiendo que no se logrará unificar lo ideológico como componente subyacente de la lectura de mundo de los actores. Por ejemplo en Chile se formó una "Comisión Nacional

10 La distinción que habría que hacer entre la acción de Trabajo Social y la de "las mesas" es que estas últimas han sido constituidas por el gobierno, y forman parte de instancias consultivas propias de cuestiones de política pública y no constituyen un mandato para el gobierno. En la acción del Trabajo Social se crean espacios democráticos en que se tratan cuestiones relativas a "lo problemático" a cualquier nivel, de la cual surgen acciones que se tienden a autoestructurar como consecuencia de compromisos libres de sujetos sociales con voluntad de articulación. 
para la Superación de la Pobreza", adoptando un eje discursivo de centro/ derecha interpretando la estructura discursiva de los grupos sociales a que se buscaba convocar, los empresarios, profesionales y organizaciones sociales, en aras de trascender las divisiones de clase.

Reconocer la existencia de discursos reticulados (diversos y en relación dinámica diferenciada), también nos remite a la búsqueda simultánea de nodos discursivos, de puntos de relación, de aspectos compartidos desde los cuales iniciar la intervención, pero no se trata de una teoría del consenso como proceso de acción, sino, de dilucidar las constricciones del medio a determinados discursos, los mecanismos de poder que cristalizan un "orden de discurso" y que operan como obstáculo a nuevas perspectivas. Se trata de situarse en "la intersección", más acordes a una distribución abierta para definir y abordar "lo problemático social". Tras este enfoque se reconoce la necesidad de generar conversaciones que recojan las diversas sensibilidades y generen procesos convergentes los que no estarán exentos de tensión. Se trata en otras palabras de acceder a los "topos o topoi", a esos referentes de sentido compartidos por una cultura desde los cuales anclar la acción social profesional.

¿Cómo actuar sobre las representaciones, o sobre las estructuras de interpretación de la realidad?, deconstruyendo, reconstruyendo, pero, cómo deconstruir. Normalmentehabiendodeveladoen el procesodeinvestigaciónlasinterpretaciones (implícitas y explícitas) con que, cada sujeto (individual o colectivo) problematiza la situación en cuestión, se trabaja con procesos de devolución y contrastación de perspectivas, es decir se crean espacios de compartir y discutir, generando conversaciones que permitan nuevas "fusiones de horizontes" entre los diversos discursos presentes en lo problemático. Este proceso no es homologable a la resolución de problemas, mediación o negociación, sino, a uno de reconstrucción colectiva en el ámbito problemático que si bien tiene algo de todos los procesos nombrados se tipifica por ser una reconfiguración del sentido y el significado de lo problemático con miras a asumirlo reflexivamente.

La creación de espacios es, de por si, un proceso técnico complejo, están involucrados temas de poder, historias de relaciones y percepciones cristalizadas que es necesario interpelar a fin de generar recomposición discursiva. Implica vulnerabilizar visiones y posiciones particulares presentadas como legítimas bajo el argumento de la obviedad y la suposición de universalidad, para constituir nuevas condiciones de posibilidad, generando nuevos ordenes discursivos, es decir, actuar sobre las superficies donde se mantienen y hegemonizan determinadas maneras de definir 
lo problemático y sus soluciones. En otras palabras, se trata de crear condiciones de posibilidad más que realizar o persuadir directamente, esto implica un cierto grado de pensamiento creativo estratégico que evite caer en los lugares comunes de la imposibilidad instalada en las prácticas cotidianas. Por ejemplo, si se quiere cambiar la relación entre profesores y estudiantes frente al proceso pedagógico, es necesario no caer en los circuitos de descalificación mutuas, asumiendo lo que dicen los estudiantes, "que el profesor no enseña y nos trata mal", o lo que dicen los profesores, "que a los estudiantes no les interesa aprender", sino generar un escenario en que estos se vean compelidos a actuar cooperativamente en actividades de aprendizaje fuera del aula, rompiendo con la verticalidad y la estructuración rígida de los espacios, generando recursos para actividades extra aula y dando responsabilidades de organización a los estudiantes. Lo mismo puede ser pensado respecto la situación de niños en la calle, cuando se generan entornos de apoyo a las actividades cotidianas de estos y no se les saca de la calle simplemente para protegerlos institucionalizadamente. Se trata de estudiar "las condiciones de posibilidad" que cada escenario posee y facilitar "entornos generadores", escenarios creativos a partir de las propias realidades de los sujetos.

En Chile tenemos algunos ejemplos de este tipo de acciones que, aun cuando reflejan éxito sólo relativo, ilustran a nivel macrosocial lo propuesto, uno de ellos, de extrema complejidad, fue la mesa de diálogo respecto la creación de medidas de reparación y cooperación con la justicia en casos de violación de derechos humanos ocurridas durante y a propósito del Gobierno Militar. Las "mesas"11 como metodología reflejan en parte esta perspectiva, y aunque obedecen a un orden institucional de gran escala, no son muy diferentes de los esfuerzos de cientos de Trabajadores Sociales en la dirección de estructurar redes, y redes de redes en los procesos de intervención locales.

Qué es una red sino un intento de coordinar acciones, conversaciones, visiones en un territorio y un tema. En todo intento de articulación de actores están presentes los procesos de iteración discursiva y diferenciación, sea en una población, en una familia, en una comuna, o el país.

En un nivel más profundo de desarrollo esta aproximación interventiva implica generar condiciones que permitan endogeneizar los procesos, generando actores, sujetos capaces de autodiagnóstico, autoaprendizaje, autocuidado, autodesarrollo. En algunos casos esta orientación está disminuida estructuralmente, como en el caso de problemas mentales de base física ${ }^{12} \mathrm{o}$ en algunos cuadros clínicos mentales en que la capacidad de "darse cuenta" está

11 La distinción que habría que hacer entre la acción de Trabajo Social y la de "las mesas" es que estas últimas han sido constituidas por el gobierno, y forman parte de instancias consultivas propias de cuestiones de política pública y no constituyen un mandato para el gobierno. En la acción del Trabajo Social se crean espacios democráticos en que se tratan cuestiones relativas a "lo problemático" a cualquier nivel, de la cual surgen acciones que se tienden a autoestructurar como consecuencia de compromisos libres de sujetos sociales con voluntad de articulación.

12 Aunque se ha demostrado que, aún en casos de concomitancia de cierto patrón genético en determinados tipos de conducta, no es más que un concomitancia mediada, es decir la presencia de un patrón genético no determina la aparición de una conducta si no encuentra en el entorno condiciones para su desarrollo. Esto es parte delosúltimos desarrollos de la "epigenética" disciplina que estudia la presencia de factores concomitantes al desarrollo de condiciones biogenéticas originarias. 
13 En el sentido que a este concepto dan Berger y Luckman en "La Construcción Social de la Realidad". Editorial Herder, Madrid, 1993.

${ }^{14}$ Del autor ver: Mind, Self and Society. Charles W. Morris (ed). Chicago: University of Chicago Press. S.M.D.

15 A propósito del autor revisar: El Problema de los Géneros Discursivos. Siglo XXI, México, 1989.

16 Vygotsky, Lev. El Desarrollo de los Procesos Psicológicos Superiores. Ed. Grijalbo. México, 1988. alterada. No obstante, siempre éste será un límite de baja frecuencia y pueden ser más o menos claramente delimitados.

Así como hemos dado relevancia, hasta ahora, a la dimensión simbólica en que se expresan procesos materiales y estructurales sociales, no podemos dejar de pensar que la acción profesional abarca las bases materiales que constituyen dialécticamente el sustento y efecto de los mundos representacionales que definen las situaciones problemáticas socialmente construidas.

\section{La Dialéctica entre lo Material/Estructural y lo Simbólico}

Los mundos representacionales se constituyen a partir de la experiencia histórica, desde los espacios cotidianos, desde el "mundo inmediato". ${ }^{13}$ Esta base experiencial tiene referentes estructurales y materiales evidentes en los que descansa la dialéctica simbólico/material. Por ejemplo la vivienda, a más de implicar la satisfacción de una necesidad material de protección, cumple una función simbólica, implica un orden externo y otro interno, la vida interna discurre mediante la apropiación de espacios por parte de los integrantes de la familia, un mundo de significados se va construyendo en relación a cada lugar del hogar. La vida externa de la casa esta referida al barrio y la apariencia externa de la construcción, pero la visión de la familia también remite a un grupo social de origen que posee una posición en la estructura. No sólo hay que tener una buena vivienda, sino vivir en un buen barrio y pertenecer a un grupo social de cierta preeminencia para acceder a una plena inserción social. La inserción social es material y simbólica, no basta con una casa, no basta con un buen barrio, también implica un nivel cultural y de remuneraciones, así, la intervención implica distintos órdenes que van relacionándose unos con otros. Bien sabido es el nivel de estigmatización a que son sometidas ciertas poblaciones y grupos a partir de la imagen que se ha construido socialmente de estos, al punto que buena parte de las preocupaciones actuales de estos sectores sociales está centrada en posicionar la "verdadera imagen de lo que son", en un mundo que ha depreciado su valía y ha ejercido violencia simbólica.

Tanto George Herbert Mead ${ }^{14}$ desde el interaccionismo simbólico, como más particularmente Mijail Bajtín ${ }^{15}$ desde la polifonía del discurso, y Lev Vigotzky ${ }^{16}$ desde el construccionismo, ponen de manifiesto la relación entre la praxis material situada y la construcción de lo que hemos nombrado como "estructuras de interpretación". Los procesos de socialización familiar, escolar, laboral y social global se constituyen así en un eje vertebrador de la manera como los sujetos 
se ven a sí mismos, construyen subjetividad y se relacionan con los otros. Lo importante de esta consideración es la mediación social y estructural que está presente en la manera como los sujetos se constituyen.

Las identidades locales, personales, sociales de clase o grupo son constituidos por mediaciones presentes en los procesos de socialización de los que no están ausentes los medios de comunicación, los pares etáreos o sociales, y los grupos de afecto de que los sujetos participan en cada etapa de la vida familiar, laboral o social.

El reconocimiento de una base material / estructural implica para la intervención la acción sobre factores estructurados, esto es, respecto a las posiciones y el poder de los sujetos en los procesos que definen los contenidos y la orientación de las normas y las instituciones en el nivel sociopolítico, así como también, respecto a las dinámicas psicosociales y socioculturales que configuran su vida cotidiana.

Dicho de esta forma, pareciera proponerse una cierta dualidad entre la materialidad e inmaterialidad de los procesos. Es necesario señalar que esta polarización es sólo con pretensión didáctica y heurística con el fin de discernir mejor los procesos en que se interviene y su doble movimiento constitutivo. Tampoco es posible afirmar polarmente lo material y lo inmaterial como cuestiones claramente discernibles, es evidente que existe una mutua implicación y una gradación desde lo más material a lo más inmaterial que hace difícil leer esta relación como dos caras distintas siendo ambas de una misma moneda.

Las implicancias que esta consideración tiene para la intervención es que no se puede romper la tensión material / inmaterial en la intervención. A ratos pareciera que algunas acciones de Trabajo Social quisieran prescindir de lo simbólico y se anclan en lugares cercanos al asistencialismo o al condicionamiento operante prescindiendo de la dimensión material estructural, situando lo problemático en las condiciones materiales existenciales, aislando los eventos de su totalidad comprensiva.

Por otra parte, hay otro tipo de acciones que tienden a sobredimensionar la perspectiva simbólica pensando que al resignificar la forma como se piensa sobre un fenómeno este podría ser cambiado, sin considerar las constricciones materiales estructurales. 


\section{La Dimensión Ético/Política como Transformación de las Condicionantes Simbólico/Materiales que Limitan la Conciencia de las Relaciones Sociales y Espaciales}

Si bien, una parte de la condición material y estructural no está al alcance inmediato de la intervención de Trabajo Social por constituir dimensiones relacionadas con las estructuras económicas o normativas globales, es posible considerar como vector de intervención ético/política, el desarrollo de sujetos de acción cada vez más conscientes de sus relaciones con lo estructural y de los mundos simbólicos de que son portadores. Esto nos remite a la toma de conciencia de la historicidad personal y social, que se desplaza desde la visión de mundo referida a lo individual/familiar/grupal y por tanto, a la acción en los ámbitos privados, a la comprensión del sujeto como parte de una estructura social más amplia y por tanto concibiéndose como sujeto colectivo, como sujeto de derechos más allá de su ámbito privado, para finalmente pasar a la condición de sujeto que asume su potencial de creador de sociedad, constructor consciente de su propio destino como sujeto político.

Tejer estas relaciones desde el eje ético/político, nos inmuniza respecto a un estilo de acción en lógica de redes de tipo sistémica que tiende a prescindir de la necesidad de articulación de identidades en torno a las cuales puedan crecer acciones conjuntas de mejoramiento del entorno con un sentido de historicidad, con perspectivas de proyección de algo que somos y no de algo que nos viene dispuesto, constituido por la historia de acoplamientos estructurales en la comunicación a partir de la oferta de coordinación de acciones para fines del mercado o el Estado.

Más que una red es necesario articular sentidos compartidos, más que sólo respuestas a propuestas del sistema, articulación a partir del mundo de la vida más que integración sólo instrumental. Esto es así respecto a la creación de ciudadanía de las familias en su entorno poblacional o en el edifico, frente a los servicios públicos, frente a las políticas sociales.

Este eje puede ser considerado como una progresión en la autoconciencia de sujetoría de derechos como así en la progresión actorial individual, social y político colectiva.

De este modo, el vector socio / político imbrica dialécticamente a los sujetos en el movimiento que se desplaza al interior de un orden discursivo, poblado de 
representaciones diversas de lo social en tanto perspectivas biográficas, psico sociales y socioculturales, en tránsito desde los espacios privados a los públicos. El desplazamiento hacia la sujetoría de derechos y hacia una actoría política, implica una visión integrada de la acción profesional, que articula, modalidades de acción discursiva, como también gestión administrativa, gestión política, y acción educativa.

Lo que integra el proceso de intervención es evidentemente el eje ético / político, puesto que marca la orientación global del proceso. Este entiende a los sujetos individuales y sociales como seres cada vez más auto - concientes de su posición en el mundo de las relaciones sociales y espaciales, al punto de concebirse como sujeto de su propia historia, en transacción con el contexto en una disposición consciente, intencionada y reflexiva.

Este proceso, de apropiación consciente y reflexiva de la propia historia nos ubica en una constelación que hace posible la dilucidación de los sujetos en la trama social, sujetos enfrentados a resolver sus necesidades bio - psico - sociales en un contexto histórico que se interpreta con toda su riqueza y diversidad.

Podemos hacer una distinción respecto a esta progresión en la constitución de actores en dos coordenadas, una referida a la progresión de la disposición a la acción que va del orden implicado, al orden asumido activo, al orden co - creable y otra referida a los niveles de acción frente a lo problemático social.

\section{LA INTERVENCIÓN COMO PROGRESIÓN}

\begin{tabular}{|l|l|l|l|}
\hline \multicolumn{1}{|c|}{ Disposición } & $\begin{array}{l}1 \text { Orden } \\
\text { implicado }\end{array}$ & $\begin{array}{l}2 \text { Orden } \\
\text { asumido activo }\end{array}$ & $\begin{array}{l}3 \text { Orden } \\
\text { co - creable }\end{array}$ \\
\hline ORDEN DISCURSO & Asumido reflejamente & $\begin{array}{l}\text { Como proceso asu- } \\
\text { mido activamente }\end{array}$ & $\begin{array}{l}\text { Como sujeto de inter- } \\
\text { vención }\end{array}$ \\
\hline $\begin{array}{l}\text { ESTRUCTURAS DE } \\
\text { INTERPRETACIÓN }\end{array}$ & $\begin{array}{l}\text { Como visión natural de } \\
\text { las cosas }\end{array}$ & $\begin{array}{l}\text { Como interpretacio- } \\
\text { nes particulares del } \\
\text { universo social }\end{array}$ & $\begin{array}{l}\text { Deconstrucción, recon- } \\
\text { strucción de representa- } \\
\text { ciones }\end{array}$ \\
\hline SOCIO/POLİTICO & $\begin{array}{l}\text { Individual } \\
\text { Familiar Privado } \\
\text { Adaptación a un orden }\end{array}$ & $\begin{array}{l}\text { Social como Público } \\
\text { Sociedad Civil } \\
\text { Dilucidación de prácti- } \\
\text { cas hegemónicas }\end{array}$ & $\begin{array}{l}\text { Lo político colectivo } \\
\text { Proyecto de sociedad } \\
\text { Articulación de sentidos }\end{array}$ \\
\hline
\end{tabular}

El orden del discurso es a las estructuras de interpretación y la gestión sociopolítica, el sustrato desde el que las tecnologías de constitución de subjetividad operan, es el referente desde el que se persuade a partir del mercado de bienes y político. Los mensajes remiten a lo socialmente establecido, a los discursos compartidos. De 
17 Gadamer, Hans. "Verdad y Método". Ed. Sígueme. Salamanca. 1977. P. 337.

18 Proceso cercano al propuesto como "darse cuenta" en el discurso de la Gestalt. El concepto de metacognición pertenece a la descripción de procesos cognitivos desde el congnitivismo y se usa para referir el proceso de autoobservación que un sujeto puede realizar del acto que realiza en el momento que lo ejecuta, sea este de tipo mental o material. allí que sea vital la capacidad de transitar hacia las estructuras de interpretación, puesto que es la forma como se organizan los procesos de captura de "lo real" por los sujetos, las que han sido aprendidas a partir de la socialización mediática, y la educación. Las estructuras de representación se constituyen a partir de los libretos sociales que se van incorporando. Asumir la existencia de estos libretos permite reconocer la presencia en la forma de organizar la realidad de una historia de aprendizajes. El nivel socio/político es el referente potencial activo para la transformación de las condiciones simbólicas y materiales en que se constituye la subjetividad y la objetividad de la existencia. Constituye el espacio más relevante dado que implica la orientación de la acción bajo una perspectiva integrada en un proyecto de sociedad. El referente sociopolítico supone la dilucidación paralela de los niveles discursivos y representacionales puesto que de lo contrario, la condición de posibilidad de una acción conciente e intencionada estaría limitada. Sin dilucidar como se organiza la realidad no se puede intervenir desde lo discursivo como medio para la deconstrucción/ reconstrucción, pero es en el lenguaje donde se refleja la manera como se organiza la realidad.

Si damos una mirada a estos niveles en cuanto su progresión, en el ámbito discursivo, se distingue un momento de "orden implicado", en que la conciencia de la existencia de un orden discursivo y de un potencial de acción desde el discurso están aún latentes, se asume la acción discursiva como producción - reproducción sin plena conciencia del lenguaje como el ámbito en el que nos constituimos y desde el que construimos sociedad. En el segundo momento de orden asumido - activo, se produce la percepción de que lo que reproducimos forma parte de un orden discursivo, tomamos distancia de este y podemos pasar a la fase de orden co - creable, en tanto cuanto sabemos que en el discurso se "fusionan horizontes", se constituyen "nuevas realidades". Cada vez que conversamos, la realidad deja de ser de unos o de otros, sino se estructura en lo que está "entre" nosotros, construyéndose mientras compartimos. Sabemos que no podemos sino participar de ese proceso, no podemos dejar de interpretar desde nuestro horizonte el horizonte del otro, y desde allí proponer nuestro decir en busca de interlocución interpretativa entendiendo finalmente con Gadamer que "El ser que puede ser comprendido es el lenguaje". ${ }^{17}$

La progresión de las estructuras de interpretación apunta, ya no a la conciencia de ser parte de un orden discursivo y de poder actuar en la construcción de este, sino, a la posibilidad de descentrarse y hacer el ejercicio metacognitivo ${ }^{18}$ de mirar nuestros propios procesos de organización de "la realidad" y asumir su carácter particular respecto otras formas de hacerlo, permitiendo en este proceso esclarecer 
los límites y posibilidades de nuestras estructuras de interpretación con miras a su reorganización.

La progresión de lo socio político, por su parte, dice relación a la historicidad, a la potencialidad individual, grupal y colectiva de hacerse cargo de nuestra situación en el mundo, de participar en la constitución de lo social. La progresión implica ir de la adaptación a un cierto orden establecido, a la dilucidación de las formas de constitución de las hegemonías, para terminar en la capacidad de articular sentidos para generar proyectos colectivos. En este sentido se asume la propuesta de Habermas ${ }^{19}$ apelando a la creación de condiciones contrafácticas para el desarrollo de actos de habla libres, no otra cosa se estructura con la creación de "espacios" participativos.

A modo de conclusión, enumeraremos las implicancias más relevantes que posee esta forma de aproximarse conceptualmente a la intervención:

a) Se entiende la acción profesional como educida a partir de la trama de relaciones que se constituye a propósito de un interés compartido. Pero educida de manera reflexiva, intencionada y consciente, en tanto, cuanto Trabajo Social posee intencionalidades derivadas de su ser ético / político.

b) La acción se organiza en función de la creación y ampliación de condiciones de posibilidad, en el desarrollo de pensamiento estratégico de segundo orden. No es ya la acción directa sobre un problema, sino la acción sobre las condiciones que lo reproducen, no en un sentido meramente estructural sociopolítico, sino, sociocultural y psicosocial.

c) Desde otra perspectiva, el cambio surge plausiblemente desde la práctica. Se crea condiciones de hacer las cosas de otro modo. Experimentar puede estar antes del cambio, cambiar un escenario, genera un movimiento reflexivo que va de la acción, al sentimiento y la reflexión. Se trata de una invitación a la osadía de reinventar condiciones de deconstrucción de problemas, más que la confrontación directa con los nudos no resueltos.

d) Las coordenadas de comprensión y acción reflejan para nuestra intención al interior del ideario moderno, el ir a un sujeto consciente de la trama de sus relaciones sociales en la búsqueda de dilucidar sus propios presupuestos

\footnotetext{
${ }^{19}$ Habermas, J. Pensamiento postmetafísico". Madrid. 1990. P. 85.
} 
representacionales, capaz de definir su "ser en el mundo" con cada vez mayor autonomía y sentido de pertenencia solidaria, con mayor sentido de su ser histórico.

e) Un desplazamiento de la acción desde los espacios privados a la apropiación de los espacios públicos, en la perspectiva del reconocimiento de lo que la acción colectiva puede significar para el perfeccionamiento de la vida en común, la democracia y por su intermedio a la conformación de un nuevo orden de relaciones sociales.

f) En este nuevo orden de relaciones (de sujetos conscientes articulados democráticamente), se puede pensar que los beneficios del progreso material (tan acariciado por el ideario moderno) pueden ser crecientemente accesibles a todos, esto potenciado por la fuerza del creciente reconocimiento de derechos políticos, sociales y culturales que propugnan las formas humanizadoras del orden global que en lo normativo se esta buscando construir.

\section{BIBLIOGRAFIA}

CALSAMIGLIA, H. Y Tusón, A. (2002) Las cosas del decir. Manual de análisis del discurso. Editorial Ariel. Barcelona.

ECHEVERRÍA, R. (2004) Ontología del lenguaje. Editorial Océano. Santiago de Chile.

FAIRCLOUGH, N. (1989) Language and Power. Longman Group U.K. Limited. London/ N.Y.

FOUCAUIT, M. (1991) Arqueología del Saber. Ed. Siglo XXI. Méjico.

GADAMER, H. (1977) Verdad y Método I y II. Ed. Sígueme. Salamanca.

HABERMAS, J. (1990) Pensamiento postmetafísico. Madrid.

MOLITOR, M. Le hermenéutique collective, en "Méthodes d'analyse de contenu et sociologie". Facultés Universitaires Saint-Louis. Bruxelles, 1990. Pág. 23 - 31.-

ONETO, L. (2003). "La Representación de la Pobreza en los Textos de los Centros de Estudio del Chile de los Noventa". Tesis para optar al Grado de Doctor en Lingüística. Universidad Católica Valparaíso.

PARRET, H. (1993) Semiótica y Pragmática. Edicial S.A..Buenos Aires.

SARANGI, S. And SLEMBROUCK, S. (1996) Language, Bureaucracy \& Social Control. Longman. London / New York.

TITCHER, S.,MEYER, M., WODAK, R. And VETTER, E. (2000) Methods of Text and Discourse Analysis. Sage Publications, London/New Delhi. 
VASILACHIS DE GIALDINO, I. (1998) Discurso político y prensa escrita. Gedisa editorial. Barcelona.

WODAK, R. y MEYER, M. (2003) Métodos de análisis crítico del discurso. Ed. Gedisa. Barcelona.

BIOGRAFIA

LEONARDO ONETO PIAZZE

Trabajador Social, profesor de la Escuela de Trabajo Social de la Pontificia Universidad Católica de Valparaíso, Chile. Master en Trabajo Social de la Universidad de Puerto Rico y Doctor en Lingüística de la Pontificia Universidad Católica de Valparaíso. (loneto@ucv.cl) 